\title{
A Blinded Randomized Controlled Trial Evaluating the Usefulness of a Novel Diet (Aminoprotect Care) in Dogs with Spontaneous Food Allergy
}

\author{
Thierry OLIVRY ${ }^{1)}$, Keigo KURATA ${ }^{1)}$, Judy S PAPS ${ }^{1)}$ and Kenichi MASUDA ${ }^{1)}$ \\ ${ }^{1)}$ Center for Comparative Medicine and Translational Research, College of Veterinary Medicine, NC State University, Raleigh, North \\ Carolina, 27606, U.S.A.
}

(Received 16 April 2007/Accepted 12 June 2007)

\begin{abstract}
Aminoprotect Care (APC) is a novel diet composed of aminoacids, potato proteins and corn starch. The objectives of this study were to determine whether Maltese-Beagle atopic (MBA) dogs hypersensitive to corn exhibited clinical signs and changes in immunological markers after being fed APC. The study was designed as a blinded randomized controlled crossover experiment. Ten MBA dogs with signs of allergy within five days of ingesting corn were selected. Dogs were randomized to be fed either their maintenance diet with corn or APC for five days. After a washout of two weeks, diets were switched. Before and daily during each intervention, skin lesions were graded by an investigator while pruritus was assessed by another. Before and at the end of each intervention, the percentage of circulating $\mathrm{CD} 4^{+} \mathrm{CCR} 4^{+}$, corn-activated $\mathrm{CD} 4^{+} \mathrm{T}$-lymphocytes and serum corn-specific IgE levels were measured and ratios of post:pre values calculated. During this trial, pruritus and skin lesions increased significantly in MBA dogs when ingesting corn while no such increase occurred when fed APC. Total, median and maximal pruritus values were significantly higher in MBA dogs ingesting corn compared to APC. There were no significant differences between interventions in the immunological parameters assessed. In summary, even though APC contains corn starch to which corn-sensitive MBA dogs often react, the ingestion of APC did not lead to significant increases in skin lesions or pruritus. Aminoprotect Care might prove valuable for management of food allergies. These experimental observations must be validated in large field studies.

KEY WORDS: atopy, canine, food allergy, hypoallergenic diet.
\end{abstract}

In dogs, common causes of skin inflammation, pruritus and secondary skin lesions include allergic, infectious and parasitic diseases. Among allergies, clinicians must differentiate between environmental, food and ectoparasite (e.g. fleas) flare factors. Such diagnostic challenge might be difficult because of the common coexistence of hypersensitivity to multiple allergens $[4,15]$. To determine whether cutaneous lesions or pruritus are due, in part or in full, to dietary components, food trials are performed for a duration of four to ten weeks [16]. Improvement of signs during such trial is suggestive of an adverse food reaction, but confirmation of diagnosis must await the demonstration of recurrence of cutaneous and/or digestive signs after rechallenge with the patient's former diet [16]. As an aid to the diagnosis of adverse food reactions, commercial diets are available, which contain either novel or hydrolyzed proteins [16]. The hydrolysis of proteins into smaller peptides is expected to reduce - at least theoretically — peptide binding to allergenspecific IgE, thereby potentially reducing their allergic potency [16].

Aminoprotect Care (APC; Nosan Corporation, TsukubaCity, Ibaraki, Japan) is a novel reduced allergenicity diet whose main ingredients consist of aminoacids, potato proteins and corn starch. It is proposed for use in dogs sus-

\footnotetext{
* Correspondence to: Olivry, T., Department of Clinical Sciences, North Carolina State University, College of Veterinary Medicine, Research Building, 4700 Hillsborough Street, Raleigh, NC 27606, U.S.A.

e-mail: Thierry_Olivry@ncsu.edu
}

pected of being affected with food allergies. Preliminary studies have suggested the absent in vitro reactivity of serum IgE and peripheral lymphocytes from dogs with food allergy to extracts made from this diet [10]. Moreover, a pilot clinical trial confirmed that signs abated by more than $50 \%$ in four dogs with food allergies fed Aminoprotect Care [10].

North Carolina State University's Maltese-Beagle Atopic (MBA) dogs have been shown to develop spontaneous hypersensitivity to food ingredients fed previously [6]. In a previous study, 14 MBA dogs showed clinical signs of skin allergy following the ingestion of $200 \mathrm{mg} / \mathrm{kg}$ of corn (10 dogs) and/or soy (11 dogs) [7]. Remarkably, one third of the dogs that reacted to corn also exhibited cutaneous reactivity after the intake of a similar amount of corn starch [7].

The purpose of this study was to determine, using a randomized blinded controlled trial design, whether the administration of APC triggered relapses of clinical signs and changes in relevant biological markers in MBA dogs, a canine model of food-induced atopic skin lesions. Results suggest that the ingestion of APC by MBA dogs with spontaneous corn-allergy is not followed by relapses of either cutaneous or digestive signs by at least $90 \%$ of subjects, nor is it associated with significant changes in tested biological markers of allergy. As a result, APC may prove beneficial in the diagnosis or management of food allergy in dogs. The usefulness of this novel diet must be verified in a field trial enrolling outbred dogs with pruritus suspected of being of allergic origin. 


\section{MATERIAL AND METHODS}

This study was designed as a blinded randomized controlled crossover experiment with ten study subjects and lasting 4 weeks. This number of dogs was selected so that the trial had a $80 \%$ power to detect a $30 \%$ difference in clinical sign severity after each phase (i.e. intervention) of the study (standard deviation $=30 \% ; \mathrm{P}=0.05$ ) [8]. All aspects of this study were approved beforehand by NC State's Institutional Animal Care and Use Committee (IACUC).

Selection of study subjects: Fifteen MBA dogs were fed their routine maintenance diet (Eukanuba KO, Iams, Dayton, OH, U.S.A.), a diet containing kangaroo meat and oat flour. In addition to their routine diet, all dogs received 400 $\mathrm{mg} / \mathrm{kg}$ of cracked corn each morning, and they were monitored five days per week for any development of pruritusassessed by the observation of scratching, biting, chewing, rubbing - and/or erythema. The administration of corn was discontinued after one week or after the development of clinical signs above, whichever occurred first. Ten MBA dogs that exhibited the highest severity of erythema and pruritus within five days of beginning ingesting corn were selected for the clinical trial phase of the study.

Clinical trial: Each of the ten MBA dogs was randomized, by coin toss, to receive either its maintenance diet (i.e. Eukanuba KO) along with $400 \mathrm{mg} / \mathrm{kg}$ of cracked corn (positive control) or APC (test diet) twice daily for five days. After a washout of two weeks in which all dogs received the maintenance diet, there was crossover and the dogs received the intervention not fed before (Fig. 1). The number and identity of dogs assigned to each intervention was kept separately by individuals feeding the animals so that both clinical evaluators remained blinded to the diet fed during both phases of the trial. The nature and order of the interventions was revealed to investigators only at the end of the trial.

Before and once daily during each experimental phasebut not during the washout periods - the extent and severity of pruritus manifestations and skin lesions were assessed independently by 2 different investigators.

To evaluate the severity of pruritic manifestations, a 2dimensional composite pruritus visual analog scale (PVAS) was designed. This scale consisted of a $100 \mathrm{~mm}$ wide square - with lines every millimeter - with pruritus "duration" graded on the $\mathrm{x}$ axis and "intensity" graded on the $\mathrm{y}$ axis. The investigator marked a dot at the intersection corresponding to the subjective evaluation of both duration and intensity of pruritic manifestation each morning for $30 \mathrm{~min}$, $1 \mathrm{hr}$ after dogs were fed. After recording the $\mathrm{x}$ and $\mathrm{y}$ coordinates of each dot, the PVAS was determined with the following formula: PVAS = x.y/100, or, in other words, this score was equal to one hundredth of the product of duration and intensity of pruritic manifestations observed during 30 $\min$.

To grade skin lesions, a lesional scale (LS) derived from the third version of the validated CADESI was used [14]. At each of 20 different body sites, the severity of 4 skin lesions (erythema, papules, excoriations and self-induced alopecia) was assessed using a grade of 0 (none) to 5 (severe), as for the CADESI-03 [14]. While the first 2 primary lesions are representative of previous acute and subacute flares, the last two were secondary lesions that occur after pruritic selftrauma. Altogether, these body sites and skin lesions were selected as they had been observed previously after allergen challenge in MBA dogs. The maximum LS value achievable therefore was 20 sites $\times 4$ lesions $\times 5$ grades $=400$ units.

Finally, the presence of vomiting, diarrhea or soft stools was assessed on a daily basis during each intervention.

Clinical outcome measures: At the end of the study, and
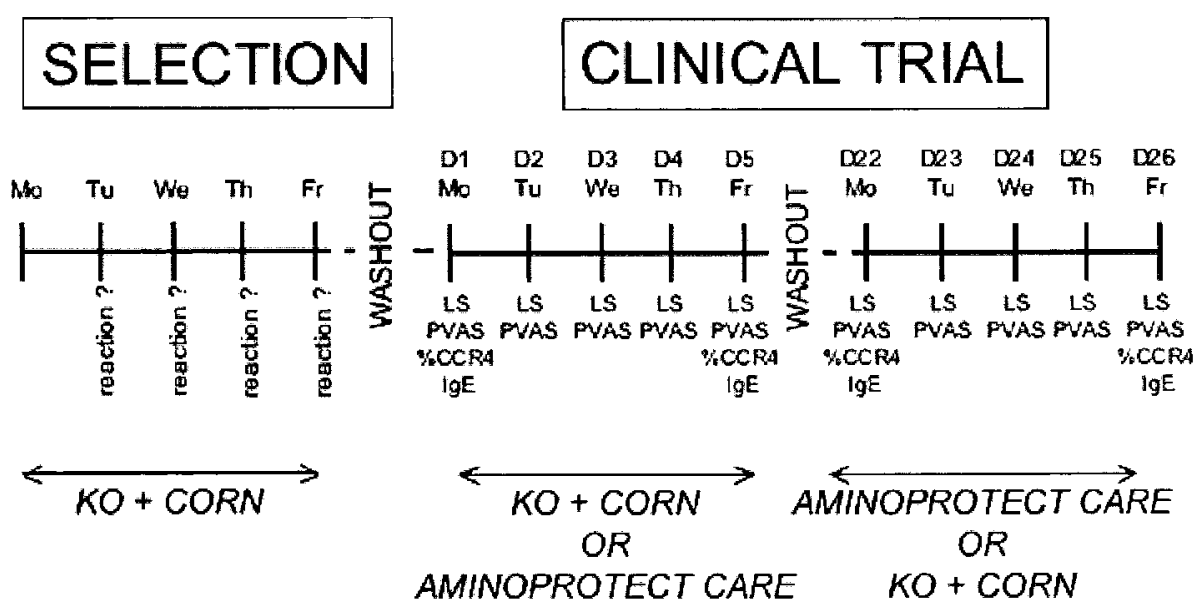

Fig. 1. Study Diagram: After a selection phase, ten corn-reacting MBA dogs were randomized to be fed either an active control diet of $\mathrm{KO}$ and corn or the Aminoprotect Care test diet for 5 days. After a washout of 2 weeks, interventions were reversed during a crossover. Before and once daily during each phase of the trial, the degree of pruritus and skin lesions were graded by two different investigators blinded to the intervention. Before and at the end of each intervention, blood was collected for determination of percentage of circulating CCR4 $4^{+}$helper T-lymphocytes and serum corn-specific IgE. 
after the sequence of intervention was revealed for each dog, the following parameters were calculated for each phase $(\mathrm{KO}+$ corn or APC) and for each subject:

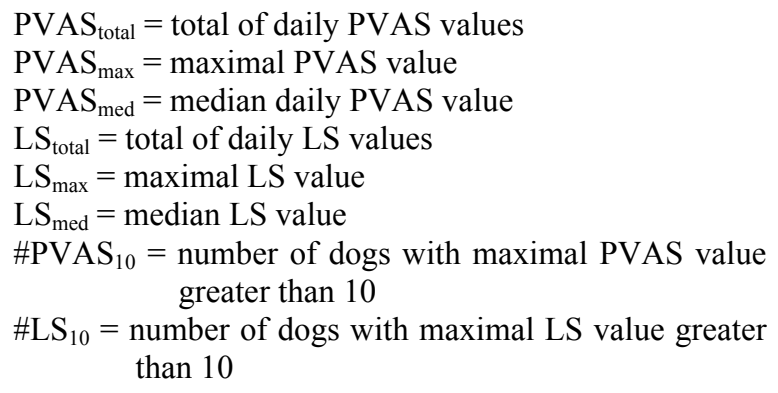

Before beginning, and on the afternoon of the last day of each interventional phase, blood was collected from each subject for determination of three immunological parameters.

Determination of circulating CCR4-positive Helper Tlymphocytes: Purification of peripheral blood mononuclear cells (PBMCs) from whole blood and flow cytometry for $\mathrm{CD} 4{ }^{+} \mathrm{CCR} 4^{+}$cell population were performed as reported previously with slight modifications [9]. Briefly, whole blood samples were obtained from MBA dogs using EDTAtreated collection tube. Peripheral blood mononuclear cells (PBMCs) were purified from whole blood using FicollHypaque (Nycomed Pharma AS, Oslo, Norway) and incubated with phosphate-buffered saline containing 5\% mouse serum (Jackson ImmunoResearch Laboratories, Inc, PA, U.S.A.) at $4^{\circ} \mathrm{C}$ for $15 \mathrm{~min}$, followed by staining with Alexa 647-labeled anti-canine CD4 (Serotec Ltd, Oxford, UK) and PE-labeled anti-human CCR4 monoclonal antibodies (BD Pharmingen, San Diego, CA, U.S.A.). Alexa 647-labeled purified rat IgG2a (Serotec LtD) and PE-labeled mouse IgG1 (Serotec Ltd) were used as negative controls. Propidium Iodide (BD Pharmingen) - positive cells were gated out to exclude dead cells on this analysis. On gating of the lymphocyte fraction, the proportion of $\mathrm{CD} 4^{+} \mathrm{CCR} 4^{+}$cells in $\mathrm{CD}^{+}$cells was determined by BD LSR II (BD Biosciences, San Jose, CA, U.S.A.). Data were analyzed using DiVa software (BD Biosciences). Finally, the ratios of $\mathrm{CD} 4{ }^{+} \mathrm{CCR} 4^{+}$cell percentages before and after exposure to each diet were calculated for each dog and compared between groups.

Determination of activated helper T-lymphocytes after in vitro stimulation with corn antigen: Peripheral blood mononuclear cells were cultured in RPMI 1640 medium containing $10 \%$ fetal bovine serum and antibiotics $(100 \mu \mathrm{g} / \mathrm{m} l$ of streptomycin and $100 \mathrm{U} / \mathrm{m} l$ of penicillin) with corn antigen at a concentration of $10 \mu \mathrm{g} / \mathrm{m} l$ together with $5 n \mathrm{~g} / \mathrm{ml}$ of human recombinant IL-2 (PeproTech Inc., NJ, U.S.A.) at $37^{\circ} \mathrm{C}$ under $5 \% \mathrm{CO}_{2}$ in air for 6 days. This duration of cell culture was determined in a pilot study. Cells stimulated with phytohemagglutinin (PHA) at $5 \mu \mathrm{g} / \mathrm{m} l$ were also used as positive controls. As described above, cells were stained with Alexa 647-labeled anti-canine CD4 antibody (Serotec
Ltd, Oxford, UK) and PE-labeled anti-human CD25 antibody (DAKO A/S, Denmark), and then the percentages of double positive cells in a relatively large $\mathrm{CD}^{+}$lymphocyte population were analyzed using LSRII and DiVa software. A stimulation index between samples cultured with corn antigen and medium alone was calculated (corn/medium). As determined above, the ratios of stimulation indices between before and after exposure to each diet were expressed in each dog and compared between interventions.

Determination of serum corn-specific IgE levels: The level of corn-specific IgE was measured by a fluorometric ELISA as reported previously with slight modifications [11]. Briefly, microplates were coated with corn antigen extract (Greer Laboratories, Lenoir, NC) diluted with 0.05 $\mathrm{M}$ carbonate buffer at a dilution of 1:250. After blocking with PBS containing $10 \%$ fetal bovine serum, sera from MBA dogs diluted with the blocking buffer at a dilution of 1:5 were added to each well and incubated at room temperature for $3 \mathrm{hr}$. A pool of sera from ten specific-pathogenfree dogs was used as negative control, while sera from dogs with known high IgE reactivity to corn were used as positive controls. The plates were then incubated with biotinylated mouse anti-dog IgE (Clone; 5.91) that was kindly provided by Dr. Hammerberg (North Carolina State University) [3] at $4{ }^{\circ} \mathrm{C}$ overnight. Finally, streptavidin-beta-galactosidaseconjugate (Roche Diagnostics GmbH, Penzberg, Germany) and $0.1 \mathrm{mM} 4$-methylumbelliferyl-D-galactoside (Sigma Chemical Co., MO, U.S.A.) were used as an enzymatic reaction which was stopped with $0.1 \mathrm{M}$ glycine- $\mathrm{NaOH}(\mathrm{pH}$ 10.2 ) after $2 \mathrm{hr}$ incubation at $37^{\circ} \mathrm{C}$. The fluorescence intensity was read as fluorescence units (FU) on Fluoroskan Acent FL (Thermo Fisher Scientific, Inc, Waltham, MA).

Statistical analyses: Clinical and laboratory parameters were compared between active control ( $\mathrm{KO}+$ corn) and test (APC) interventions using either two-tailed nonparametric repeated measures t-tests (Wilcoxon signed rank tests; parameters 1 to 6 ) or Fisher's tests (parameters 7 and 8). Similarly, baseline and maximal PVAS and LS values were compared, within each intervention, using the same Wilcoxon signed rank tests. All statistical analyses were performed using a statistics software (Prism 4.0 for Mac, Graphpad, San Diego, CA, U.S.A.), and the threshold for significance was assessed at $5 \%(\mathrm{P}=0.05)$.

\section{RESULTS}

Study subjects: Of the 15 MBA dogs challenged with 400 $\mathrm{mg} / \mathrm{kg}$ of cracked corn, ten manifested pruritus, with or without erythema, within five days of introduction of this allergen to which they had reacted in the past. The ten MBA dogs selected in this study consisted of three males and seven females aged from 3 to 9 years (median: 8.5 years).

Assessment of pruritus: When MBA dogs were fed the active control diet of KO and corn, PVAS values increased significantly while such values remained at low levels in nine of ten dogs when fed APC (Fig. 2). In eight dogs eating corn, maximal PVAS values exceeded 10, while this bench- 


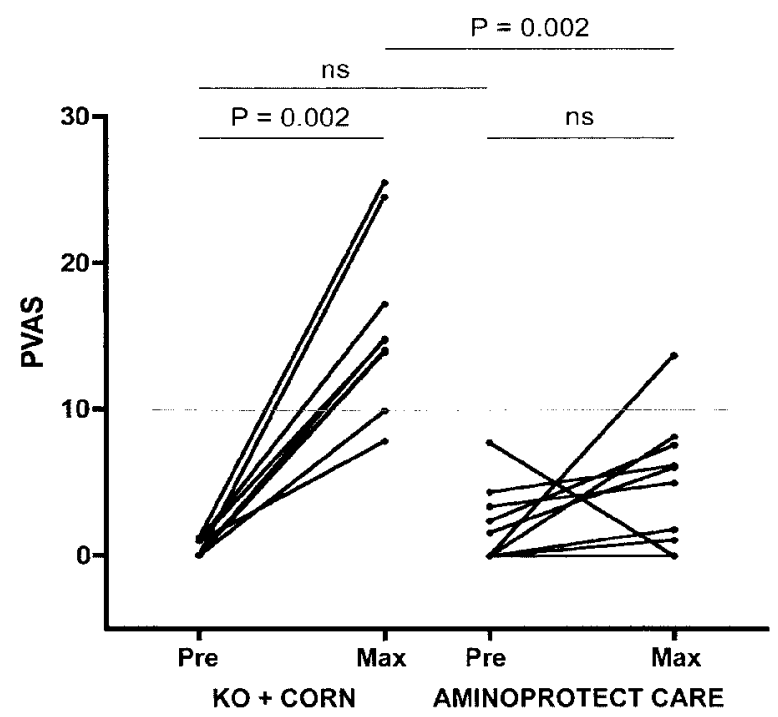

Fig. 2. Evolution of Pruritus Manifestations: Line graph of baseline (pre) and maximal (max) PVAS values for each dog during both intervention phase. Pruritus values increased significantly when corn was ingested, but the change was not significant (ns) when dogs were fed the Aminoprotect Care test diet. After five days, maximal PVAS values were significantly higher in dogs eating corn than in those ingesting the test diet.

mark was exceeded only by one dog ingesting APC (Fisher's test; P 0.0055). Total (Fig. 3), maximal and median daily PVAS values were significantly higher during the ingestion of KO and corn compared to APC (Table 1).

Assessment of skin lesions: After beginning eating KO and corn, LS values increased significantly, while this did not occur in dogs fed APC (Fig. 4). Two and zero dogs had maximal LS greater than 10 when fed the active and test diets, respectively (Fisher's test; $\mathrm{P}=0.4737$ ). Of note is that, during none of the two interventions were maximal LS in excess of 20, an indication that dogs developed at best very mild skin lesions. Total (Fig. 5), maximal and median daily LS values were not significantly different during the administration of $\mathrm{KO}$ and corn compared to APC (Table 1).

During this study, none of the MBA dogs enrolled exhibited vomiting, diarrhea or soft stools after being fed either the active control or test diets.

Determination of circulating CCR4-positive helper Tlymphocytes: The percentage of peripheral helper $\mathrm{CD} 4^{+}$

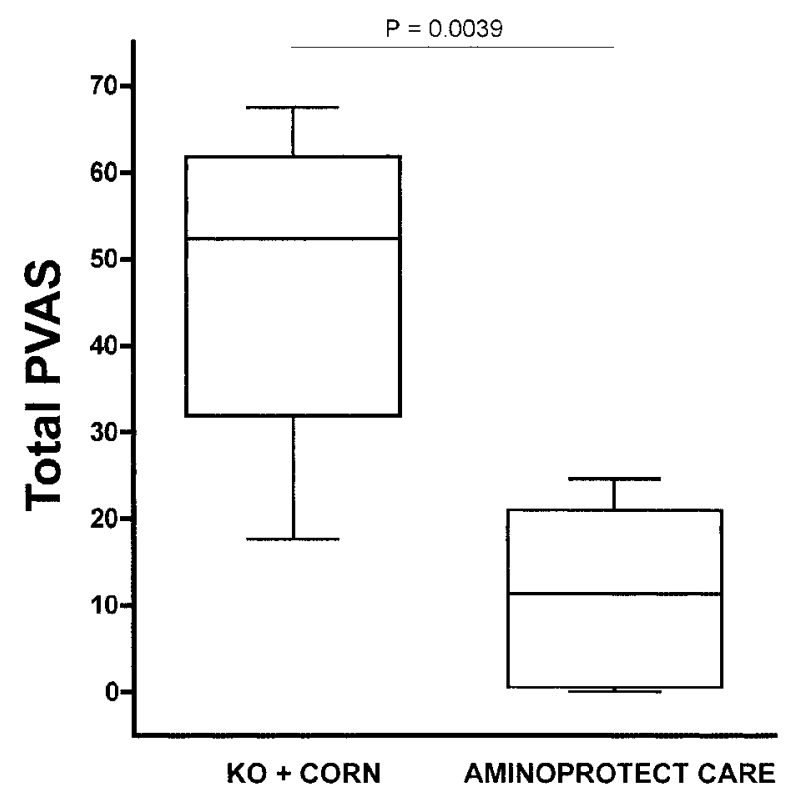

Fig. 3. Total Pruritus Scores: Box-and-whisker plot of the total PVAS values during each intervention. Total PVAS values were significantly higher in dogs fed the active compared to the test diets. Box $=$ interquartile range; bar $=$ median; whiskers $=$ range.

helper T-lymphocytes expressing CCR4 did not change markedly during either phase of the study. Ratios of changes in percentages of $\mathrm{CD} 4^{+} \mathrm{CCR} 4^{+}$cells (post divided by pre values; Table 2) were not significantly different between active and test dietary interventions even though values were higher after the active diet compared to APC in seven of ten dogs.

Determination of activated helper T-lymphocytes after in vitro stimulation with corn antigen: The ratios of stimulation indices between after and before exposure to each diet did not change remarkably during the study (Table 2). Comparison of values obtained for each dog challenged with each intervention neared significance, however, with ratios calculated after the active diet being higher than those after APC in eight of ten dogs.

Assessment of serum corn-specific IgE: Serum levels of corn-specific IgE did not increase or decrease significantly during either intervention. Untransformed values (i.e. fluorometric units) were not significantly different between

Table 1. Clinical outcome measures

\begin{tabular}{lccccc}
\hline \multirow{2}{*}{ Parameter } & \multicolumn{2}{c}{ Active Diet (KO + Corn) } & \multicolumn{2}{c}{ Test Diet (APC) } & Active vs. Test \\
& median & $95 \% \mathrm{CI}$ & median & $95 \% \mathrm{CI}$ & P value \\
\hline 1. PVAS $_{\text {total }}$ & 52.4 & $35.1-59.4$ & 11.3 & $3.9-18.9$ & 0.0039 \\
2. PVAS $_{\text {max }}$ & 14.7 & $11.7-19.7$ & 5.6 & $1.8-8.1$ & 0.0020 \\
3. PVAS $_{\text {med }}$ & 10.5 & $7.2-13.0$ & 1.3 & $0.3-2.9$ & 0.0039 \\
\hline 4. $\mathrm{LS}_{\text {total }}$ & 13.5 & $6.3-20.9$ & 4.5 & $3.3-13.2$ & 0.1055 \\
5. $\mathrm{LS}_{\text {max }}$ & 5.5 & $3.7-9.9$ & 3 & $2.6-6.8$ & 0.1309 \\
6. $\mathrm{LS}_{\text {med }}$ & 2.5 & $1.0-4.9$ & 0.8 & $0.3-3.1$ & 0.1641 \\
\hline
\end{tabular}




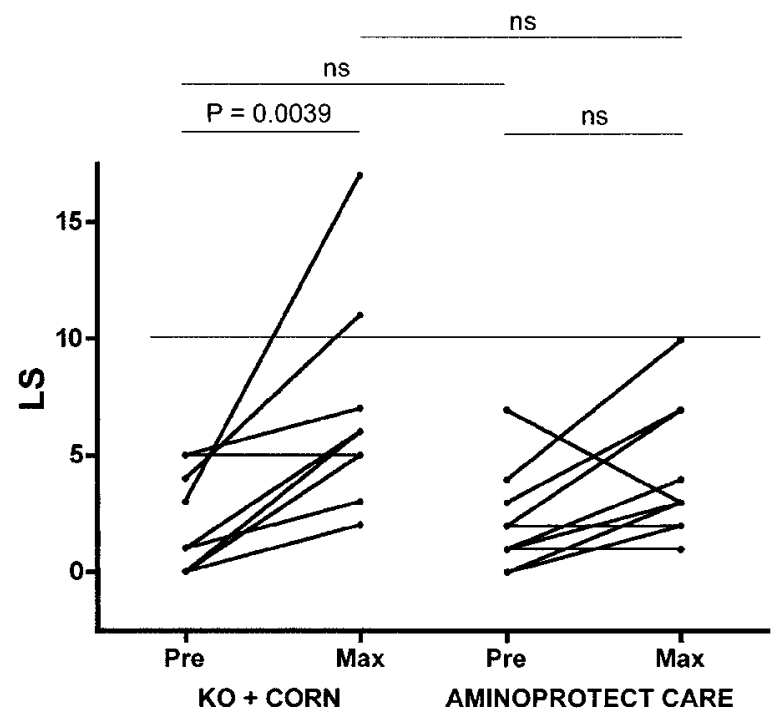

Fig. 4. Evolution of Skin Lesions: Line graph of baseline (pre) and maximal $(\max )$ LS values for each dog during both interventions. Skin lesion scores increased significantly when corn was ingested, but the evolution was not significant (ns) when dogs ate Aminoprotect Care.

groups before or after administering active and test diets (data not shown). Similarly, ratios of values obtained after and before each intervention were not significantly different between active and test groups (Table 2 ).

\section{DISCUSSION}

In this short term blinded randomized controlled crossover trial, the ingestion of APC - a novel reduced allergenicity diet - did not trigger clinical signs of allergy in nine of ten MBA dogs with spontaneous allergy to corn, while the administration of the culprit allergen exacerbated pruritus, with or without skin lesions in all of these dogs with food-induced atopic dermatitis. However, the duration of each food challenge was insufficient to detect any variation in examined cellular (percentage of $\mathrm{CD} 4{ }^{+} \mathrm{CCR} 4^{+}$and activated $\mathrm{CD}^{+} \mathrm{T}$-lymphocytes) and specific humoral (cornspecific IgE) immune responses.

North Carolina State's MBA dogs are unique in their spontaneous development of pruritus, atopic-like skin lesions, otitis plus or minus colitis in response to the ingestion of food items to which they have been exposed earlier [6]. As a result, dogs in this colony have been used for testing of the allergenic potential of novel diets including hydrolysates [7]. In a previous study, $14 \mathrm{MBA}$ dogs were

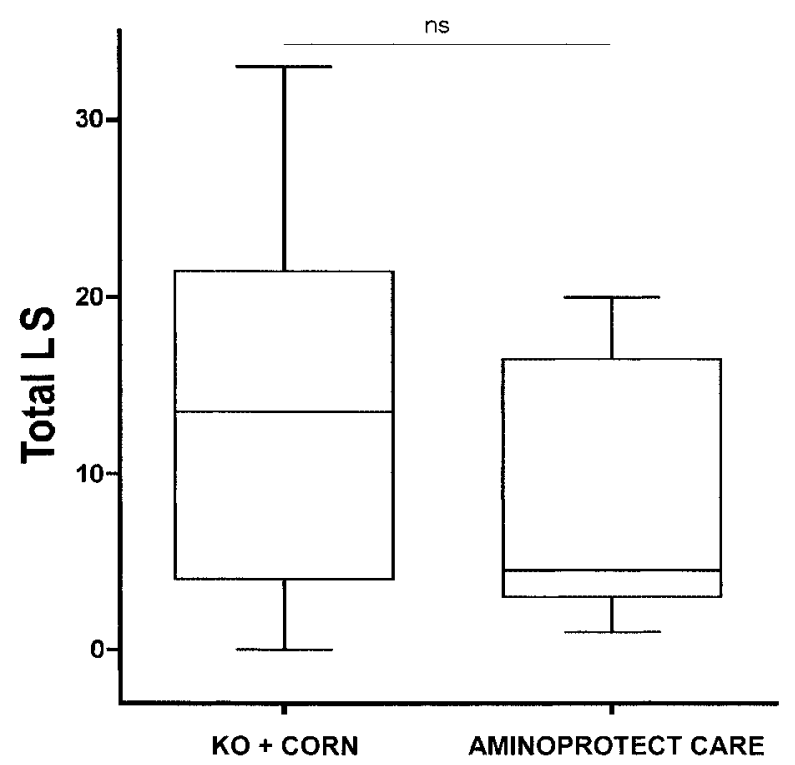

Fig. 5. Total Skin Lesion Scores: Box-and-whisker plot of the total LS values during both intervention. Total skin lesion scores were not significantly (ns) different when dogs were fed the different intervention diets. Box $=$ interquartile range; bar $=$ median; whiskers $=$ range .

challenged orally with $200 \mathrm{mg} / \mathrm{kg}$ of cornstarch, corn and soy, and three $(21 \%)$, ten $(71 \%)$ and $11(78 \%)$ of them developed adverse reactions with increases in lesional scores, respectively [7]. In all dogs, clinical signs were reported to arise within $48 \mathrm{hr}$ of food challenge [7]. In the present trial, ten MBA dogs were selected because of their development of pruritus within five days of introduction of cracked corn in their maintenance diet. After oral challenge with this allergen, pruritus scores increased within hours of ingestion, with a maximum value observed $48 \mathrm{hr}$ after challenge, as described previously [7].

In most dogs, however, the development of pruritus was not associated with a noticeable increase in lesional scores, as shown by the observation that the maximal score achieved in this trial was 17 units of a scale of 400 . This lack of erythematous eruption is best explained by the shortterm nature of the food challenges (five days), a duration that would be likely to trigger immediate, but not delayed eczematous indurated skin lesions characteristic of chronic atopic dermatitis.

In contrast, the ingestion of the test diet APC did not lead to relevant increases in either pruritus or lesional scores except for one dog who, for an unknown reason, developed transient pruritus on the first day of ingestion of APC

Table 2. Immunology parameters

\begin{tabular}{|c|c|c|c|c|c|}
\hline \multirow{2}{*}{ Post: Pre Ratios } & \multicolumn{2}{|c|}{ Active Diet (KO + Corn) } & \multicolumn{2}{|c|}{ Test Diet (APC) } & \multirow{2}{*}{$\begin{array}{c}\text { Active vs. Test } \\
\text { P value }\end{array}$} \\
\hline & median & $95 \% \mathrm{CI}$ & median & $95 \% \mathrm{CI}$ & \\
\hline $\mathrm{CD} 4^{+} \mathrm{CCR} 4^{+}$ & 1.35 & $0.75-1.37$ & 1.12 & $0.63-1.40$ & 0.4922 \\
\hline $\mathrm{CD} 4^{+} \mathrm{CD} 25^{+}$after corn & 1.48 & $1.27-1.70$ & 0.84 & $0.66-1.40$ & 0.0645 \\
\hline Corn-specific IgE & 0.93 & $0.86-1.01$ & 1.01 & $0.87-1.06$ & 0.6953 \\
\hline
\end{tabular}


$\left(\right.$ PVAS $\left._{\max }=13.8\right)$. Remarkably, scores returned spontaneously to baseline values by the next day and for the remainder of the intervention phase, while this subject was still eating APC. It is doubtful, therefore, that the noted increase in pruritus occurred secondarily to the intake of that particular diet. Of particular interest in the observation that MBA dogs did not react to this dietary challenge in spite of APC containing corn starch, an ingredient to which one third of tested MBA dogs are known to react clinically [7].

When comparing both interventions, all three outcome measures of pruritus (total, maximal and median PVAS values) were significantly higher in dogs when eating corn compared to APC. Within each intervention, maximal PVAS values were significantly higher than baseline ones only in dogs eating corn but not APC. Altogether, these results suggest that APC is of value to prevent development of the cardinal symptom of skin allergy-pruritus-in dogs with spontaneous food allergy. Of note is that none of the dogs enrolled in this trial were affected with either vomiting or diarrhea when eating each of the diets.

To assess the cellular immune response to corn allergen, we elected to determine before and after each intervention the percentage of peripheral blood lymphocytes expressing both CD4 and CCR4, a relevant marker of type-2 helper Tcells (Th2 lymphocytes) [9]. Of particular interest is that the baseline level of blood CCR4 $4^{+}$lymphocytes averaged $16 \%$ with a maximum of $43 \%$ of $\mathrm{CD}^{+}$cells in MBA dogs, a range that is typical of humans and dogs with active atopic dermatitis $[9,12,13,17]$. This finding is noteworthy as, in humans with atopic dermatitis, the percentage of peripheral helper T-lymphocytes expressing CCR4 correlates with both serum IgE levels [12, 13], lesion severity scores [13] or blood eosinophilia [12]. In this study, the two dietary challenges that lasted five days did not result in consistent changes of percentages of CCR $4^{+}$lymphocytes, or post versus pre-intervention ratios, a not surprising finding in light of the short duration of the interventions. Indeed, in a previous study, the sensitization of healthy dogs to Japanese cedar pollens resulted in an average $5 \%$ increase in CCR $4^{+}$ helper T-lymphocytes percentages, but this increase was measured one week after two sensitizing injections separated by two weeks [9].

Studying whether antigen-specific T-lymphocytes can be influenced after a dietary change in MBA dogs was of relevance, since it has been reported that lymphocyte proliferative responses in dogs with food allergies decrease with the elimination of culprit food antigens [5]. In this study, we elected to use flow cytometric analysis of activated T-lymphocyte population in $\mathrm{CD}^{+}$lymphocytes. The anti-human CD25 antibody used for this purpose has been shown to identify canine leukocytes activated with PHA [2]. In contrast, there is another report showing that this anti-human CD25 antibody did not recognize canine lymphocytes [1]. In our study, however, a large proportion of PHA-stimulated lymphocytes were identified with this reagent (data not shown). Although the specific canine molecule detected with this antibody has not been identified yet, It is conceiv- able-and likely-that activated lymphocytes could be detected by this assay. Therefore, the cell population detected with the antibody in this study should be considered to be activated $\mathrm{CD}^{+} \mathrm{T}$-lymphocytes. In this study, the ratio of stimulation indices was below 1.0 before challenge with the active diet containing corn in MBA dogs hypersensitive to corn and a population of corn-reactive CD4 ${ }^{+} \mathrm{T}-\mathrm{lym}-$ phocytes was detected after corn ingestion. This observation suggests that MBA dogs would be valuable to investigate T-lymphocyte responses to food antigens. When comparing values between control and test diets, the median ratio of corn-activated T-lymphocytes was greater after feeding the active diet compared to APC, however, there was a statistically significant difference was not detected. In a previous study using dogs with food allergies, it took at least more than three weeks of food elimination to identify a reduction in lymphocyte proliferative responses to food antigens [5]. It is hypothesized that a higher and significant reduction of T-lymphocyte activation in MBA dogs would have required a longer test period of corn avoidance using APC as an elimination diet. In addition, it is also important that this assay system to detect activation T-lymphocytes should be compared with lymphocyte proliferative responses with ${ }^{3} \mathrm{H}$-thymidine incorporation assay, since this current system could be an alternative assay to detect antigen-specific lymphocyte responses without having to use radioactive techniques.

In this study, serum corn-specific IgE levels were high in all enrolled MBA subjects compared to those of normal control dogs, an observation reported previously [7]. During both intervention phases, the levels of corn-specific IgE did not fluctuate markedly, and there were no significant differences between groups either in raw fluorometric units or post versus pre intervention ratios. Again, this observation is best explained by the short duration of the challenges with serum levels of IgE determined within five days of each other for each phase. These results suggest that serum levels of allergen-specific IgE are of little value for short duration clinical trials.

In summary, the feeding of MBA dogs-animals spontaneously predisposed to develop IgE-mediated allergy to dietary and environmental allergens-with the novel reduced allergenicity diet APC does not result in noticeable increases in pruritus and skin lesions. As a result, this diet should prove of benefit to prevent recurrence of clinical signs in client-owned animals suspected of food allergy. This hypothesis must be confirmed in a large field study.

ACKNOLWEDGEMENTS. The authors are grateful to Dr. Ken Lee (Greer laboratories, Lenoir, North Carolina) and Bruce Hammerberg (NC State University) for providing reference sera with high levels of corn-specific IgE.

Funding and Conflict of Interest:

This study was funded by the Nosan Corporation, Yokohama City, Kanagawa, Japan to NC State University, Raleigh, NC, U.S.A. One of the authors, Kenichi Masuda, 
reports having received honorarium from the sponsoring company as one of its advisory board members.

\section{REFERENCES}

1. Chabanne, L., Marchal, T., Kaplanski, C., Fournel, C., Magnol, J. P., Monier, J. C. and Rigal, D. 1994. Screening of 78 monoclonal antibodies directed against human leukocyte antigens for cross-reactivity with surface markers on canine lymphocytes. Tissue Antigens 43: 202-205.

2. Galkowska, H., Waldemar, L. O. and Wojewodzka, U. 1996. Reactivity of antibodies directed against human-antigens with surface-markers on canine leukocytes. Vet. Immunol. Immunopathol. 53: 329-334.

3. Gebhard, D., Orton, S., Edmiston, D., Nakagaki, K., DeBoer, D. and Hammerberg, B. 1995. Canine IgE monoclonal antibody specific for a filarial antigen: production by a canine $\mathrm{x}$ murine heterohybridoma using B cells from a clinically affected lymph node. Immunology 85: 429-434.

4. Hillier, A. and Griffin, C. E. 2001. The ACVD task force on canine atopic dermatitis $(\mathrm{X})$ : is there a relationship between canine atopic dermatitis and cutaneous adverse food reactions? Vet. Immunol. Immunopathol. 81: 219-226.

5. Ishida, R., Masuda, K., Kurata, K., Ohno, K. and Tsujimoto, H. 2004. Lymphocyte blastogenic responses to inciting food allergens in dogs with food hypersensitivity. J. Vet. Intern. Med. 18: $25-30$.

6. Jackson, H. A. and Hammerberg, B. 2002. Evaluation of a spontaneous canine model of immunoglobulin E-mediated food hypersensitivity: Dynamic changes in serum and fecal allergen-specific immunoglobulin $\mathrm{E}$ values relative to dietary change. Comp. Med. 52: 316-321.

7. Jackson, H. A., Jackson, M. W., Coblentz, L. and Hammerberg, B. 2003. Evaluation of the clinical and allergen specific serum immunoglobulin $\mathrm{E}$ responses to oral challenge with cornstarch, corn, soy and a soy hydrolysate diet in dogs with spontaneous food allergy. Vet. Dermatol. 14: 181-187.

8. Lenth, R. V. 2006. Java Applets for Power and Sample Size, http://www.stat.uiowa.edu/ rlenth/Power.

9. Maeda, S., Ohmori, K., Yasuda, N., Kurata, K., Sakaguchi, M., Masuda, K., Ohno, K. and Tsujimoto, H. 2004. Increase of CC chemokine receptor 4-positive cells in the peripheral CD4(+) cells in dogs with atopic dermatitis or experimentally sensitized to Japanese cedar pollen. Clin. Exp. Allergy 34: 14671473.

10. Masuda, K., Ishihara, J., Ohmori, K., Kaneko, M., Hatano, Y. and Watanabe, K. 2005. Pilot studies of a novel prescription diet, Aminoprotect Care, as an elimination diet for the diagnosis of canine food hypersensitivity (abstract). Vet. Dermatol. 16: 204.

11. Masuda, K., Tsujimoto, H., Fujiwara, S., Kurata, K., Hasegawa, A., Taniguchi, Y., Yamashita, K., Yasueda, H., DeBoer, D. J., deWeck, A. L. and Sakaguchi, M. 2000. IgE-reactivity to major Japanese cedar (Cryptomeria japonica) pollen allergens (Cry j 1 and Cry j 2) by ELISA in dogs with atopic dermatitis. Vet. Immunol. Immunopathol. 74: 263-270.

12. Nakatani, T., Kaburagi, Y., Shimada, Y., Inaoki, M., Takehara, K., Mukaida, N. and Sato, S. 2001. CCR4(+) memory CD4(+) $\mathrm{T}$ lymphocytes are increased in peripheral blood and lesional skin from patients with atopic dermatitis. J. Allergy Clin. Immunol. 107: 353-358.

13. Okazaki, H., Kakurai, M., Hirata, D., Sato, H., Kamimura, T., Onai, N., Matsushima, K., Nakagawa, H., Kano, S. and Minota, S. 2002. Characterization of chemokine receptor expression and cytokine production in circulating $\mathrm{CD} 4^{+} \mathrm{T}$ cells from patients with atopic dermatitis: up-regulation of C-C chemokine receptor 4 in atopic dermatitis. Clin. Exp. Allergy 32: $1236-1242$.

14. Olivry, T., Marsella, R., Iwasaki, T. and Mueller, R. 2007. Validation of CADESI-03, a severity scale for clinical trials enrolling dogs with atopic dermatitis. Vet. Dermatol. 18: 79-96.

15. Sousa, C. A. and Halliwell, R. E. W. 2001. The ACVD task force on canine atopic dermatitis (XI): the relationship between arthropod hypersensitivity and atopic dermatitis in the dog. Vet. Immunol. Immunopathol. 81: 233-238.

16. Verlinden, A., Hesta, M., Millet, S. and Janssens, G. P. J. 2006. Food allergy in dogs and cats: a review. Crit. Rev. Food Sci. Nutr. 46: 259-273.

17. Wakugawa, M., Nakamura, K., Kakinuma, T., Onai, N., Matsushima, K. and Tamaki, K. 2001. CC chemokine receptor 4 expression on peripheral blood CD4+ T cells reflects disease activity of atopic dermatitis. J. Invest. Dermatol. 117: 188196. 